Les bases libérées des protéinates l'emportent de beaucoup, comme ordre de grandeur, sur les bases liées aux acides organiques ; leur quantité est assez variable suivant les laits, soit que varie la quantité de caséine ou soit que la caséine contienne plus de bases.

Le procédé ne peut donc être employé pour le dépistage de l'alcalinisation frauduleuse du lait, ainsi qu'on le voit d'après le tableau ci-dessus. On peut tout au plus envisager que, joint au dosage de la matière grasse et du lactose, l'abaissement des chiffres indiqué par ce tableau pourrait donner un indice supplémentaire de mouillage.

\title{
DIFFICULTÉS RENCONTRÉES DANS LE DIAGNOSTIC DE LA MAMMITE STREPTOCOCCIQUE CONTAGIEUSE DES VACHES LAITIERES (1)
}

\author{
par
}

\section{COTONI, P. FORGEOT et G. THIEULIN}

Des recherches anciennes sur les divers streptocoques nous ont conduits à étudier depuis plusieurs années la mammite streptococcique des vaches laitières. L'importance économique de cette affection de nature chronique est connue dans le monde entier. Les chiffres suivants suffisent à donner une idée des pertes que cause la maladie. STABLEFORTH (2) a estimé la perte annuelle en lait de vache subie, du fait de la mammite contagieuse, en Angleterre et au Pays de Galles réunis, à 3.240.000 livres sterling. Le Dr Stwiger (3), Ministre de l'Agriculture, parlant en 1941, devant une commission du Landtag prussien, au cours de la discussion du budget de l'Agriculture, évaluait, d'après une estimation prudente, les dommages imputables à la mam mite streptococcique, à 250.000 .000 de marks-or par an.

La lecture de nombreux travaux sur le traitement, impose la notion de l'efficacité restreinte des diverses méthodes thérapeutiques proposées. Ne se heurte-t-on pas souvent au caractère invétéré des lésions? La solution du problème ne résiderait-elle pas dans l'établissement d'un diagnostic très précoce, à une époque où les signes cliniques, obscurs, incertains, absents même, ne permettent pas de reconnaître la mammite? Il n'est pas défendu d'espérer qu'on pourrait, à cette époque-là, agir efficacement, grâce à la chimiothérapie, et arrêter l'éclosion d'épidémies par un sévère isolement

(1) Soc. fr. Microbiologie, 4 novembre 1948. In Annales Institut Pasteur, 1949, $76,76$.

(2) Stableforth. Proceed. Roy. Soc, Med., 1942, 35, 625.

(3) Steiger, cité par Poreher. Le Lait, 1932, 12, n० 114, 257. 
des animaux reconnus infectés. Aujourd'hui, l'observation soigneuse des vaches d'une étable, la pratique des traites faites dans un certains ordre, répétées et totales, l'élimination des malades, semblent fournir les résultats les plus tangibles, commé l'un de nous s'est attaché, avec BEAđFRìre et GúLY (1) à le démontrer, dans les étables de la région parisienne.

Si l'on recherche la valeur des différents procédés de diagnostic de la mammite contagieuse, il est à retenir que l'ensemencement d'un lait montrant des streptocoques à l'examen microseopique direct, a pu ne nous fournir parfois aucun développement microbien, après ensemencement (2), à plus forte raison ne saurait-on compter déceler toujours le streptocoque, lorsqu'il ne serait présent dans le lait qu'à l'état de rares unités.

Que peut-on penser maintenant de l'examen du sang des vaches malades pour la recherche d'anticorps antistreptococciques éventuels ?

En France, la recherche des agglutinines dans le sang a été proposée par LeSBoU yRIÈs et AdAm (1933) (3). Mais la valeur diagnostique de ce procédé est fortement infirmée par les propriétés des streptocoques eux-mêmes, espèce bactérienne se développant gouvent à l'état agglutiné dans les milieux de culture et se montrant souvent agglutinable par les sérums d'animaux même normaux ; il faudrait, pour tirer de la méthode des résultats facilement lisibles et probants, qu'on décelât un pouvoir agglutinant vis-à-vis des streptocoques, dans le sang des vaches atteintes de mammite ; or, l'expérience ne répond pas à ce souhait. D'autre part, les échantillons de streptocoques de mammite ayant été classés en Angleterre en diverses races de constitution antigénique différente (StabLeforth) (4), il serait sans doute nécessaire de faire défiler les sérums de vaches devant l'échantillon microbien infectant ou un autre appartenant à la même race sérologique. Ces remarques rendent incertaines l'utilité de la méthode. D'ailleurs Mrnetr, Stableforth, Edwards qui ont poursuivi pendant de longues années, en Grande-Bretagne, des recherches sur la mammite, ne font eux-mêmes pas mention de la possibilité d'un séro-diagnostic agglutinant.

La recherche des précipitines peut paraître plus tentante, puisque l'agglutinabilité particulière des streptocoques cesse ici d'être un obstacle. Il est de notion courante que le sérum des lapins traités dans les veines par des injections répétées de corps microbiens

(1) Beaufrère, Gély et Thieulin. Bull. Acad. Vét. Franee, 1942, 15, no 1 ; 1943, 16, 338 ; Recueil Méd. Vét., 1946, $122, n^{\circ} 11$.

(2) Cotoni, Forgeot et Thieulin. Ces Annales, 1946, 72, 184.

(3) Lesvouyries et Adam. Bull. Acad. Vét. France, 1933, 6, 61.

(4) Stableforth. J. Path. Bact., 1937, 45, 263 ; ibid., 1938, 46, 21. 
streptococciques précipite les extraits préparés suivant la méthode de Lancefield à partir des streptocoques de même groupe et le fait se vérifie pour les streptocoques de la mammite. Mais eette propriétê précipitante a toujours fait défaut dans le sang des vaches étudié par nous. Même absence de pouvoir précipitant chez trois échantillons de sang de vaches malades, vis-à-vis d'un extrait préparé avec des streptocoques de la mammite suivant la technique de Besredka (dessiceation des microbes et broyage avec $\mathrm{NaCl}$ anhydre) ; pareil extrait avait été utilisé jadis dans des recherches sur l'immunité antistreptococcique (1). Même absence encore de pouvoir précipitant du sang de vache malade vis-à-vis de deux autres extraits, que nous remercions MM. GRABAR et OUdIN d'avoir préparés pour nous, l'un de nature polysaccharidique, J'autre à partir ds streptocoques ultrasonnés.

Des essais de détection d'antigène streptococcique dans le lait des vaches malades, au moyen de sérums antistreptococciques de lapins, ne nous ont fourni que des résultats négatifs.

Dans une direction différente, nous avons été amenés à nous demander si le sang des vaches malades possède un pouvoir antifibrinolytique vis-à-vis des streptocoques de la maladie. TrLLETT et GATENER (2) ont mis autrefois en lumière le pouvoir fibrinolytique exercé sur la fibrine humaine par de nombreux échantillons de streptocoque. Ce pouvoir sur la fibrine humaine est d'ailleurs beaucoup plus répandu parmi les streptocoques humains (28 sur 28) que parmi les streptocoques animaux (3 sur 18). Dans un travail ultérieur, Triletr (3) signale comme non fibrinolytiques, 31 échantillons de streptocoques isolés du lait et dans la mammite. D'après Tilletr (4), $75 \%$ des sujets guéris d'une infection streptococcique (scarlatine, érysipèle, amygdalite), possèdent dans le sang une antifibrinolysine. Mais la plupart des éehantillons pathogènes des mammites, appartenant au groupe B de Lancefield, paraissent dénués de pouvoir hémolytique et de pouvoir fibrinolytique (TILLETT) (5) ; aussi ne peut-on guère compter déceler régulièrement de pouvoir antifibrinolytique dans le sang de vaches malades. En fait, il nous est arrivé une seule fois de troụver un échantillon de sang de vache, présentant un pouvoir antifibrinolytique indiscutable vis-à-vis de la fibrine bovine; nous avons appris plus tard qu'il provenai d'une vache atteinte de mammite confirmée.

Il convient, ajoutons-le, de préciser à quelle espèce animale

(1) Cotoni, Césari et Chambrin. Ces Annales, 1933, 50, 608.

(2) Tillett et Garnier. Journal Exp. Med., 1933, 58, 485.

(3) Tillett. Journal Bact., 1935, 29, 111.

(4) Tillett. Journal clin. Invest., 1935, 14, 276.

(5) Tillett. Bact. Rev, 1938, 2, 161. 
appartient la fibrine utilisée dans la réaction. Les fibrines de diverses espèces animales semblent offrir, en présence d'une fibrinolysine donnée, une sensibilité inégale. Nos recherches ont été faites avec la fibrine bovine, mais même vis-à-vis de cette dernière, le pouvoir fibrinolytique des streptocoques du lait de vache se montre inconstant. Smith, Hankinson et Mudge (1) trouvent parmi 22 échantillons de streptocoques hémolytiques de lait de vache, 9 seulement capables d'attaquer la fribrine bovine ( 2 de vaches saines, 7 de vaches atteintes de mammite). En résumé, il nous paraît possible que le sang de certaines vaches malades possède un pouvoir antifibrinolytique, mais on ne saurait affirmer que ce pouvoir se rencontre toujours, même quand l'échantillon microbien infectant lyse la fibrine. Comme cette propriété semble exceptionnelle parmi les streptocoques de la mammite, la méthode en question ne semble pas susceptible d'une large application.

Enfin, dans une direction encore différente, nous avons recherché si le sérum des vaches malades, ajouté aux milieux de culture, est capable de modifier les caractères du streptocoque pathogène. Du bouillon est additionné de sérum de vache malade (1/10) et ensemencé avec le streptocoque de la mammite. Après quatre passages, la culture peut se montrer plus abondante, plus agglutinée, composée de chaînettes microbiennes notablement plus longues que la culture en bouillon-sérum de vache saine. Dans ces deux séries, nous n'avons pas réussi à mettré en lumière de différences dans les colonies obtenues sur milieux solides, et ces recherches gagneraient à être étendues.

En conclusion, presque tous nos essais aboutissent à des résultats négatifs : difficultés techniques et causes d'erreur dans la recherche du pouvoir agglutinant des sérums de vaches, pouvoir précipitant nul dans les conditions présentes, pouvoir antifibrinolytique probablement exceptionnel, modifications peu caractéristiques des streptocoques cultivés en présence des sérums pathologiques. On est donc réduit à la recherche des streptocoques par ensemencement du lait, avec les incertitudes des méthodes actuelles.

(1) Smith, Hankinson et Mudge, Proc. Soc, exp. Biol., Med., 1936, 34, 266. 